\title{
Long non-coding RNA TUG1 sponges microRNA-9 to protect podocytes from high glucose-induced apoptosis and mitochondrial dysfunction via SIRT1 upregulation
}

\author{
MIN LEI, GUIBAO KE, YAN WANG, DAN LUO and YAO HU \\ Department of Nephrology, Affiliated Hospital and Clinical Medical College of Chengdu University, \\ Jinniu, Chengdu, Sichuan 610081, P.R. China
}

Received April 1, 2021; Accepted September 16, 2021

DOI: $10.3892 / \mathrm{etm} .2022 .11161$

\begin{abstract}
Podocyte apoptosis and mitochondrial dysfunction serve a major role in diabetic nephropathy progression. The present study revealed a molecular mechanism regulating podocyte apoptosis and mitochondrial dysfunction. In vitro models were established using conditionally immortalized mouse podocyte clonal cells treated with high glucose (HG). Reverse quantitative-transcription PCR were used to detect gene expression, western blotting and immunofluorescence were used to detect protein expression, Cell Counting Kit-8 was used to detect cell viability and flow cytometry was used to detect cell apoptosis. HG treatment in the mouse podocyte clonal cells downregulated taurine-upregulated gene 1 (TUG1) expression and decreased viability in a dose-dependent manner. In addition, TUG1 knockdown (KD) increased HG-induced apoptosis, while TUG1 overexpression (OE) reduced HG-induced apoptosis in podocytes. HG-induced mitochondrial dysfunction was identified in podocytes, with increased reactive oxygen species levels, decreased complex I/III activity and decreased basal/maximal oxygen consumption rate. TUG1 KD worsened HG-induced mitochondrial dysfunction, and TUG1 OE reversed these effects. At the molecular level, TUG1 was revealed to promote sirtuin 1 (SIRT1) expression by sponging microRNA (miR)-9, and SIRT1 OE reversed the HG-induced apoptosis and mitochondrial dysfunction increased by TUG1 KD. The present data indicated that downregulation of TUG1 induced by HG was associated with HG-induced apoptosis and mitochondrial dysfunction in podocytes, and that TUG1 protected
\end{abstract}

Correspondence to: Dr Yao Hu, Department of Nephrology, Affiliated Hospital and Clinical Medical College of Chengdu University, 82 North Section 2, 2nd Ring Road, Jinniu, Chengdu, Sichuan 610081, P.R. China.

E-mail: westchinast@126.com

Key words: podocytes, apoptosis, mitochondrial dysfunction, high glucose
HG-induced podocytes by promoting SIRT1 expression via miR-9 inhibition.

\section{Introduction}

Diabetic kidney disease (DKD), the most serious microvascular complication of diabetes mellitus, is a chronic progressive kidney disease characterized by persistent proteinuria and progressive proteinuria (1). DKD is accompanied by a continuous decline in renal small glomerular filtration rate, renal capillaries rupture, and damage in glomerular capillaries and tubular interstitium (1). Podocytes, also known as upper glomerular cells, are highly terminally differentiated cells that constitute the last barrier of the glomerular filtration membrane (2). In DKD, a typical damaged podocyte disease, the number of podocytes in the kidney of patients with initial DKD decreases, and this decreases even more in patients with advanced DKD (3). Therefore, podocyte damage is a key factor in initiating proteinuria and glomerulosclerosis in DKD.

Recent studies have focused on glomerular podocyte apoptosis induced by diabetes-mediated mitochondrial dysfunction $(4,5)$. Activation of NADPH oxidase and the production of oxidative molecules in mitochondria can activate animal pro-apoptotic pathways (p38 MAPK and caspase-3), which ultimately leads to podocyte apoptosis (6). Lee et al (7) identified that a large amount of secreted TGF- $\beta 1$ mediates SMAD-7/p38 MAPK/caspase-3 activation, or Bax expression in mitochondria, leading to cytochrome $\mathrm{c}$ release from mitochondria and caspase- 3 activation, causing podocyte apoptosis in patients with diabetes. However, the molecular mechanisms of mitochondrial dysfunction and apoptosis in podocytes still remain unclear.

Long non-coding RNA (lncRNA) is a non-coding RNA with a length $>200$ nucleotides, and microRNA (miRNA/miR) is a class of non-coding single-stranded RNA molecules with a length of $\sim 22$ nucleotides encoded by endogenous genes (8). The present study focused on an evolutionarily conserved long intergenic non-coding RNA (lincRNA), taurine-upregulated gene 1 (TUG1), because it has been reported to regulate mitochondrial bioenergetics in diabetic nephropathy (9) and could sponge miR-9 (10). In addition, sirtuin 1 (SIRT1) has been reported as a target gene of miR-9, regulating high 
glucose (HG)-induced mitochondrial dysfunction and apoptosis in podocytes $(11,12)$. Therefore, TUG1 could theoretically regulate mitochondrial dysfunction and apoptosis through miR-9-mediated SIRT1 expression in podocytes. The present study aimed to verify TUG1-regulated mitochondrial dysfunction and apoptosis through miR-9-mediated SIRT1 expression using HG-induced apoptosis in podocytes.

\section{Materials and methods}

Cell culture and treatment. Conditionally immortalized mouse podocyte clonal cells (MPC5) were purchased from Shanghai Jihe Biotechnology Co., Ltd. (cat. no. CC-Y2125), and podocytes were propagated and maintained in RPMI-1640 medium (cat. no. 11875101; Thermo Fisher Scientific, Inc.) supplemented with 10\% FBS (cat. no. 10099141C; Thermo Fisher Scientific, Inc.) and $10 \mathrm{U} / \mathrm{ml}^{-1}$ recombinant mouse interferon- $\gamma$ (cat. no. PMC4033; Thermo Fisher Scientific, Inc.) at $33^{\circ} \mathrm{C}$ under a humidified atmosphere of $5 \% \mathrm{CO}_{2}$ for propagation, as previously described (13). Podocytes were cultured for different times $(24,48$ and $72 \mathrm{~h})$ at $33^{\circ} \mathrm{C}$ and incubated with normal glucose $(5 \mathrm{mM})$ as the control group, or exposed to $45 \mathrm{mM}$ mannitol as an osmotic pressure control. In the experimental group, podocytes were exposed to different glucose concentrations $(25,35$ and $45 \mathrm{mM})$ for the same times applied for the control groups $(14,15)$.

Cell Counting Kit-8 (CCK-8) assay. After the aforementioned treatments, the culture medium was removed and MPC5 cells were washed twice with PBS. Subsequently, $100 \mu$ l cell culture medium supplemented with $10 \mu \mathrm{l} \mathrm{CCK-8} \mathrm{solution} \mathrm{(cat.}$ no. C0038; Beyotime Institute of Biotechnology) was added, and then the cells were incubated for $2 \mathrm{~h}$. Finally, the optical density at $450 \mathrm{~nm}$ was measured to calculate the relative cell viability.

Reverse transcription-quantitative PCR (RT-qPCR). Total RNA from MPC5 cells was extracted using a FastPure Cell/Tissue Total RNA Isolation kit (cat. no. RC101-01; Vazyme Biotech Co., Ltd.). Complementary DNA was prepared using the PrimeScript ${ }^{\mathrm{TM}}$ RT Reagent kit (cat. no. RR047A; Takara Bio, Inc.) with the following temperature protocol: $37^{\circ} \mathrm{C}$ for $15 \mathrm{~min}$ and $85^{\circ} \mathrm{C}$ for $5 \mathrm{sec}$. Subsequently, $20 \mu \mathrm{l} \mathrm{qPCR}$ mix was prepared as described in the instructions of the Go Taq qPCR master mix kit (cat. no. A6001; Promega Corporation), and qPCR was carried out using SYBR-Green I and the following temperature protocol: Initial denaturation at $95^{\circ} \mathrm{C}$ for $2 \mathrm{~min}$, followed by $95^{\circ} \mathrm{C}$ for $5 \mathrm{sec}$ and $65^{\circ} \mathrm{C}$ for $30 \mathrm{sec}$ for 40 cycles. The relative gene expression was calculated using the $2^{-\Delta \Delta \mathrm{Cq}}$ method (16), with $\beta$-actin as the loading control. The primers for qPCR analysis were as follows: TUG1 forward, 5'-CTCTGGAGG TGGACGTTTTGT-3' and reverse, 5'-GTGAGTCGTGTCTC TCTTTTCTC-3'; SIRT1 forward, 5'-CAGCCGTCTCTGT GTCACAAA-3' and reverse, 5'-GCACCGAGGAACTACCT GAT-3'; miR-9 forward, 5'-ACACTCCAGCTGGGTCTTTGG TTATCTAGCT-3' and reverse, 5'-TGGTGTCGTGGAGTCG-3'; U6 forward, 5'-CTCGCTTCGGCAGCACA-3' and reverse, 5'-AACGCTTCACGAATTTGCGT-3'; and $\beta$-actin forward, 5'-AGGGAAATCGTGCGTGACAT-3' and reverse, 5'-GCC TCAGGAGTTTTGTCACCT-3'. U6 was a loading controls for miR-9, and $\beta$-actin was a loading controls for TUG1 and SIRT1.
Cell transfection. Small interfering RNA (siRNA) was used to knock down TUG1 expression. A total of $2.5 \times 10^{6}$ MPC5 cells were transfected with $50 \mathrm{nmol} / 1$ of siRNA (5'-UAACA AGUUCUAUUUUGAGCA-3', negative control for siRNA: 5'-UCCAAGUAGGAUCCCUUGAGC-3') and using Lipofectamine ${ }^{\circledR} 2000$ (Thermo Fisher Scientific, Inc.) at $33^{\circ} \mathrm{C}$ for $6 \mathrm{~h}$, and then replaced the cell cultured medium. Experiments were performed after $72 \mathrm{~h}$ of transfection. Similarly, $2.5 \times 10^{6}$ MPC5 cells were transfected with $50 \mathrm{nmol} / 1 \mathrm{mimic}$-negative control (NC; 5'-UCTTTTCAAGCGTTCAGTCCC-3'), inhibitor-NC (5'-AGCAAUGUCUACUCAUAGGA-3'), miR-9mimic (5'-UCUUUGGUUAUCUAGCUGUAUGA-3') or miR-9inhibitor (5'-AGAAACCAAUGCGACAUACU-3') using Lipofectamine ${ }^{\circledR} 2000$ at $33^{\circ} \mathrm{C}$ for $72 \mathrm{~h}$, and subsequent experiments were performed following $72 \mathrm{~h}$ of transfection. NC, inhibitor-NC, miR-9-mimic and miR-9-inhibitor were all synthesized at Shenggong Co., Ltd.

MPC5 cells were transfected as aforementioned with wild-type (WT) or mutated (MUT) versions of the 3'-untranslated region of TUG1 and SIRT1 cloned into pisCHECK2 (cat. no. 97157; Addgene, Inc.), using a Dual-Luciferase Assay kit (cat. no. D0010; Beijing Solarbio Science \& Technology Co., Ltd.) to detect luciferase activity according to the manufacturer's protocol. In brief, $72 \mathrm{~h}$ after being transfected with the NC, miR-9-mimic or miR-9-inhibitor, a recombinant pisCHECK2 plasmid was transfected into MPC5 cells at $33^{\circ} \mathrm{C}$ for $6 \mathrm{~h}$, and the cell cultured medium was replaced. Following $48 \mathrm{~h}$ of transfection, the cells were collected and the cell lysate was added. Cells were incubated on ice for $5 \mathrm{~min}$ and subsequently centrifuged at $500 \mathrm{x} \mathrm{g}$ and room temperature to collect the supernatant. A total of $20 \mu \mathrm{l}$ cell lysis supernatant was added to $100 \mu \mathrm{l}$ firefly luciferase reaction solution and $100 \mu \mathrm{l}$ Renilla luciferase reaction solution. Luciferase activity was detected within 30 min after mixing.

TUG1 and SIRT1 overexpression (OE) using adenovirus $(A A V)$ infection. Commercial AAVs were used to infect MPC5 to overexpress TUG1 or SIRT1. First, $1 \times 10^{6}$ podocytes were cultured for $12 \mathrm{~h}$ in 6-well cell culture plates. Subsequently, $1 \times 10^{10} \mathrm{PFU} / 100 \mu \mathrm{l}$ TUG1-AAV (customized; Vector Biolabs) or SIRT1-AAV (cat. no. AAV-272007; Vector Biolabs) were used to infect podocytes. Podocytes infected with an equivalent empty AAV (NC-AAV, a control AAV for TUG1-AAV and SIRT1 AAV; MOI=10) served as a negative control. At $48 \mathrm{~h}$ after infection, the cells were collected for further analysis.

Fluorescence in situ hybridization (FISH). FISH was performed as previously described (17). Following fixation with $4 \%$ paraformaldehyde for $15 \mathrm{~min}$ at room temperature, cells were treated with $0.5 \%$ Triton X-100 for 5 min at room temperature, and subsequently washed 3 times with PBS buffer. Cells were then washed twice in $70 \%$ ethanol and incubated in a series containing 85, 90 and 100\% ethanol for $3 \mathrm{~min}$ each. The hybrid mixture was prepared using $2.5 \mu \mathrm{l} 4 \mathrm{ng} / \mu \mathrm{l}$ human-derived TUG1 gene (NCBI gene ID:55000; synthesized by Genomeditech Co., Ltd.), $1 \mu 1$ 20X SSC buffer and $6.5 \mu 1$ formamide, and this was incubated at $72^{\circ} \mathrm{C}$ for $5 \mathrm{~min}$. The hybrid mixture was subsequently added into cell slices at $42^{\circ} \mathrm{C}$ overnight in the dark. After being washed with eluent buffer (50\% acetamide and $2 \mathrm{X} \mathrm{SSC}$ ) at $2^{\circ} \mathrm{C}$ for $5 \mathrm{~min}$, Alexa 
Fluor 488-digoxin antibody (1:5,000, cat. no. bs-0356R; Bioss) was added to the cells and incubated for $4 \mathrm{~h}$ at room temperature. Finally, cells were washed 3 times with PBS buffer, and then cell nuclei were counterstained with $5 \mu \mathrm{g} / \mathrm{ml}$ DAPI for $5 \mathrm{~min}$ at room temperature. All samples were analyzed by confocal microscopy.

Apoptosis analysis and detection of caspase-3 activity. Following the aforementioned treatments, MPC5 cells were collected and washed twice with cold PBS buffer. Subsequently, the Annexin V-FITC/7-AAD Apoptosis Detection Kit (cat. no. A213-01; Vazyme Biotech Co., Ltd.) was used according to the manufacturer's protocol, and apoptosis was determined with a flow cytometer (Flow-Count ${ }^{\mathrm{TM}}$; Beckman Coulter, Inc.). Results of the apoptosis analysis were analyzed using FlowJo software (V10; BD Biosciences).

Apoptosis was additionally reflected by caspase-3 activity, which was measured using the Ac-DEVD-AFC Caspase-3 Fluorogenic Substrate (cat. no. 556574; BD Pharmingen; BD Biosciences) according to the manufacturer's instructions. First, MPC5 cells were lysed using RIPA buffer (cat. no. 9806S, Cell Signaling Technology) on ice for $10 \mathrm{~min}$, centrifuged at $4^{\circ} \mathrm{C}$ at $500 \mathrm{x} \mathrm{g}$ for $10 \mathrm{~min}$, and the supernatants were collected. Protein concentration was quantified using the BCA method. A total of $50 \mu \mathrm{g}$ of proteins were pipetted and mixed with assay buffer supplemented with $10 \mathrm{mM}$ dithiothreitol. The fluorescence emission of AFC (400 nm) was measured with a SpectraMax Plus Microplate Reader (Molecular Devices, LLC).

Western blotting. Tissue/cells Protein Extraction Reagent (cat. no. 78510; Thermo Fisher Scientific, Inc.) was used to extract proteins from MPC5 cells. A BCA kit (cat. no. 23227; Thermo Fisher Scientific, Inc.) was used to determine the protein concentration. Subsequently, $50 \mu \mathrm{g}$ of proteins per lane were separated by $10 \%$ SDS-PAGE, and then transferred to PVDF membranes (cat. no. LC2002; Thermo Fisher Scientific, Inc.). The PVDF membranes were first blocked with 5\% skimmed milk powder for $1 \mathrm{~h}$ at room temperature, then probed with primary antibodies against SIRT1 (1:1,000; cat. no. ab189494; Abcam), Bax (1:500; cat. no. ab32503; Abcam), Bcl2 (1:1,000; cat. no. ab196495; Abcam) and $\beta$-actin $(1: 2,000$; cat. no. ab32503; Abcam) at $4{ }^{\circ} \mathrm{C}$ overnight. After being washed 3 times with PBS buffer, membranes were incubated with Goat Anti-Mouse IgG H\&L (HRP; 1:3,000; cat. no. ab6789; Abcam) or Goat Anti-Rabbit IgG H\&L (HRP; 1:3,000; cat. no. ab6721; Abcam) for $1 \mathrm{~h}$ at room temperature. Proteins were visualized with ECL solution (cat. no. WBKLS0100; Beijing Xinjingke Biotechnologies Co., Ltd.), followed by densitometry analysis using ImageJ 3.0 software (National Institutes of Health). $\beta$-actin was used as a loading control.

Mitochondrial superoxide indicator. A total of $0.5 \times 10^{5}$ MPC5 cells were seeded into Nunc ${ }^{\mathrm{TM}}$ Lab-Tek $^{\mathrm{TM}}$ Chambered Coverglass (cat. no. 155411; Thermo Fisher Scientific, Inc.). Following the aforementioned treatments, cell medium was replaced with fresh medium, and $200 \mathrm{nM}$ MitoSOX $^{\mathrm{TM}}$ Red Mitochondrial Superoxide Indicator (cat. no. M36008; Invitrogen; Thermo Fisher Scientific, Inc.) was added for $30 \mathrm{~min}$ at $33^{\circ} \mathrm{C}$. After being washed three times with PBS buffer, MPC5 cells were fixed with $4 \%$ paraformaldehyde for $15 \mathrm{~min}$ at room temperature followed by treatment with $0.5 \%$ Triton X-100 for $5 \mathrm{~min}$ at room temperature. Cell nuclei were counterstained with $5 \mu \mathrm{g} / \mathrm{ml}$ DAPI for $5 \mathrm{~min}$ at room temperature. Finally, all samples were analyzed using confocal microscopy.

Mitochondrial complexes activity. The Cell Mitochondria Isolation Kit (cat. no. C3601; Beyotime Institute of Biotechnology) was used to isolate mitochondria from MPC5 cells according to the manufacturer's instructions. The activities of the mitochondrial respiratory chain complexes were measured in microplates using specific kits: Complex I (cat. no. ab109721; Abcam) and complex III (cat. no. ab109905; Abcam) according to the manufacturer's instructions. The activity of the mitochondrial complexes was normalized to the concentration of mitochondrial protein.

Mitochondrial respiration monitoring. Mitochondrial oxidative phosphorylation reactions were assessed by comparing oxygen consumption rates (OCRs) measured with a Agilent Seahorse XF Cell Mito Stress Test kit (cat. no. 103010; Agilent) and an Xfe-24 Extracellular Flux Analyzer (Agilent Technologies, Inc.), as previously described (18). MPC5 cells $\left(2 \times 10^{5}\right.$ cells/well) were seeded into plates. After stimulation with $35 \mathrm{mM}$ glucose for $48 \mathrm{~h}$ at $33^{\circ} \mathrm{C}$, the cell culture medium was removed and replaced with bicarbonate-free and HEPES-free RPMI medium (pH 7.4; Invitrogen; Thermo Fisher Scientific, Inc.) supplemented with $2 \%$ FBS (cat. no. 10099141C; Invitrogen; Thermo Fisher Scientific, Inc.) for $1 \mathrm{~h}$ at $37^{\circ} \mathrm{C}$ in a $\mathrm{CO}_{2}$-free incubator. OCRs were measured under basic conditions; subsequently, according to the manufacturer's instructions, oligomycin, trifluoromethoxy carbonylcyanide phenylhydrazone and antimycin A were loaded into a sensor cartridge to produce a final concentration of $1,1.5$ and $1 \mu \mathrm{M}$, respectively. The data were analyzed using XFe software 2.6.1 (Agilent Technologies, Inc.) and normalized to the amount of protein loaded per well.

Statistical analysis. SPSS 20.0 software (IBM Corp.) was used to analyze the data. Data are presented as the mean \pm standard deviation and each experiment was repeated three times. Unpaired Student's t-test was used to compare the differences between two groups, and one-way ANOVA followed by Tukey's post hoc test was used to compare the differences among multiple groups. $\mathrm{P}<0.05$ was considered to indicate a statistically significant difference.

\section{Results}

$H G$ reduces TUG1 expression in podocytes. Viability was evaluated in MPC5 podocytes using a CCK-8 assay after incubation with different $\mathrm{HG}$ concentrations $(25,35$ and $45 \mathrm{mM})$ for different durations (24, 48 and $72 \mathrm{~h}$ ). Mannitol (45 mM) was used as an osmotic pressure control. The results indicated that, compared with the control group (normal glucose), HG significantly decreased the viability of podocytes (Fig. 1A). In addition, cell viability was decreased when the concentration of glucose added was increased, as well as when the incubation time with HG was increased. Previous studies have reported 

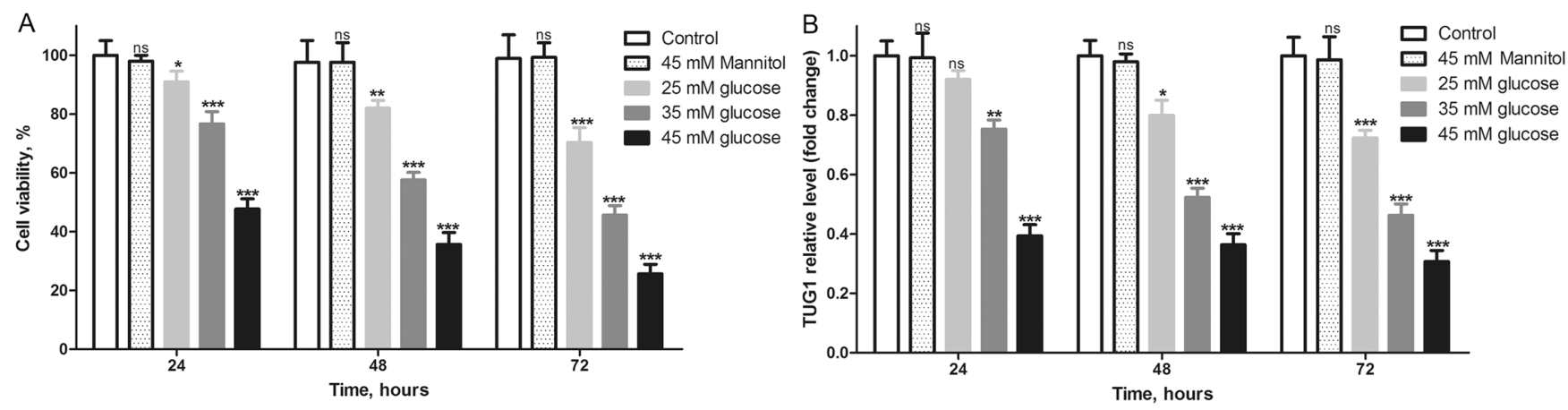

C

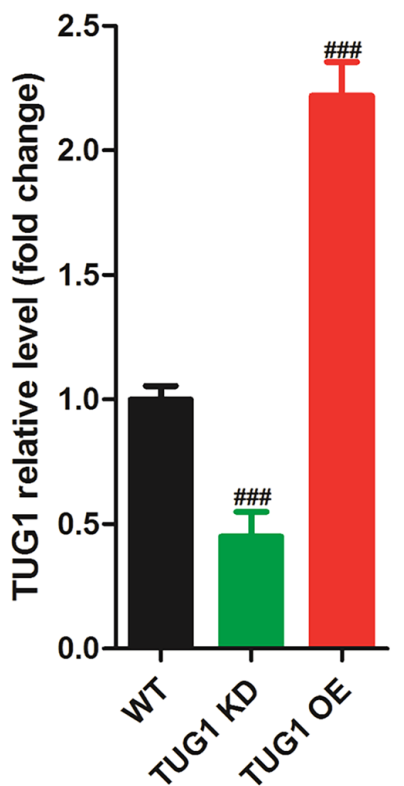

D

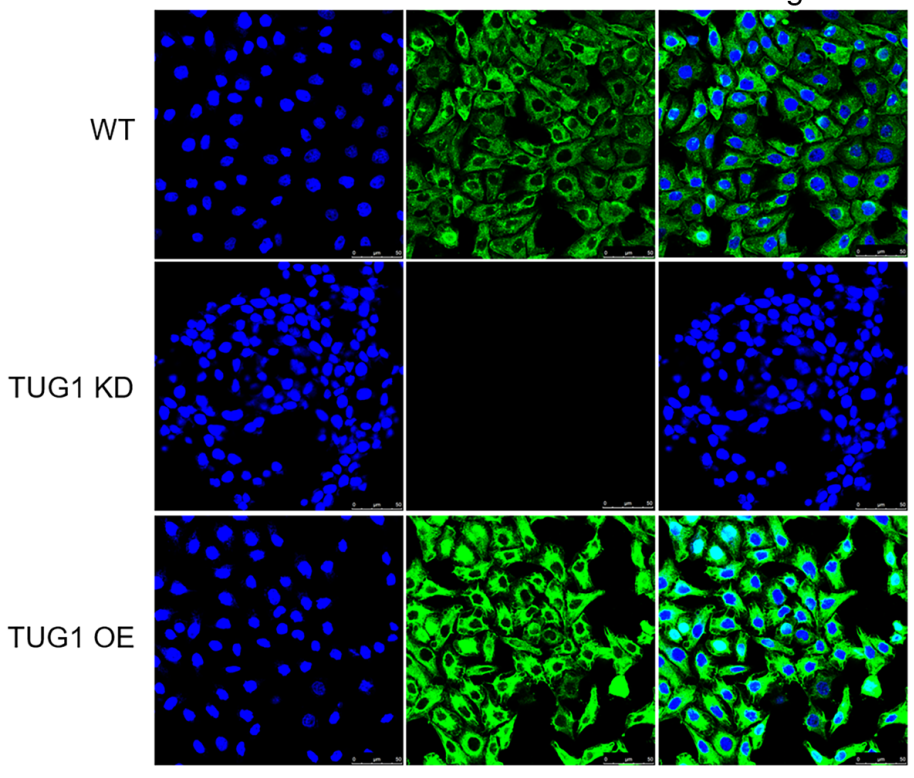

Figure 1. High glucose downregulates TUG1 expression and cell viability of mouse podocyte clonal cells in a dose-dependent manner. Following incubation with different concentrations of glucose for various durations, (A) the viability of podocytes was determined using a Cell Counting Kit-8 assay and (B) the expression levels of TUG1 were detected by RT-qPCR. (C) RT-qPCR analysis indicated that TUG1 KD and TUG1 OE were successfully established. (D) Fluorescence in situ hybridization staining revealed that TUG1 was mainly expressed in the cytoplasm and to a lesser extent in the nucleus of podocytes (magnification, x800). Each experiment was repeated at least three times, and data are presented as the mean \pm SD. P-values were calculated using one-way ANOVA followed by Tukey's post hoc test. ${ }^{\text {ns}} \mathrm{P}>0.05,{ }^{*} \mathrm{P}<0.05,{ }^{* *} \mathrm{P}<0.01$ and ${ }^{* * * *} \mathrm{P}<0.001$ vs. control group; ${ }^{\# \#} \mathrm{P}<0.001$ vs. WT group. TUG1, taurine-upregulated gene 1; KD, knockdown; OE, overexpression; WT, wild-type; RT-qPCR, reverse transcription-quantitative PCR.

that IncRNA TUG1 and podocytes serve an important role in the progression of diabetic nephropathy $(19,20)$. Therefore, it was hypothesized that HG would also affect TUG1 expression in podocytes. RT-qPCR results revealed that TUG1 expression in podocytes stimulated with $\mathrm{HG}$ was lower than that in the control group, and the higher the dose of glucose, the lower the expression level of TUG1 (Fig. 1B). Subsequently, TUG1 knockdown (KD) was established in podocytes and confirmed (Fig. S1A), and TUG1 OE was also established and confirmed (Fig. S1B). RT-qPCR analysis indicated successfully established TUG1 KD and OE podocyte cell lines (Fig. 1C). TUG1 expression in TUG1 KD podocytes was $\sim 45 \%$ of that of the WT, and its expression in TUG1 OE podocytes was $220 \%$ of that of the WT. Additionally, TUG1 expression in podocytes was observed using FISH staining, which revealed that TUG1 was expressed both in the cytoplasm and the nucleus, but mainly in the cytoplasm (Fig. 1D).

TUG1 protects podocytes from $H G$-induced apoptosis. As podocytes were treated with $35 \mathrm{mM}$ glucose for $48 \mathrm{~h}, \sim 60 \%$ of the viable cells were available for follow-up research, and HG stimulation condition was defined as $35 \mathrm{mM}$ for $48 \mathrm{~h}$ in the following experiments. After stimulation with $35 \mathrm{mM}$ glucose or $35 \mathrm{mM}$ mannitol for $48 \mathrm{~h}$, podocytes were harvested to analyze apoptosis using flow cytometry. No significant difference was observed in the ratio of apoptotic cells between the normal control and mannitol groups. However, compared with the control group, the ratio of apoptotic podocytes in the $\mathrm{WT}+\mathrm{HG}$ group was significantly increased. The percentage of apoptotic podocytes in the TUG1 KD $+\mathrm{HG}$ group was significantly higher than that in the $\mathrm{WT}+\mathrm{HG}$ group, while the percentage in the TUG1 OE + HG group was significantly lower compared with that in the WT $+\mathrm{HG}$ group (Fig. 2A and B). Subsequently, apoptosis-related indexes, such as caspase-3 activity and the expression levels of $\mathrm{Bax}$ and $\mathrm{Bcl} 2$, were evaluated. Caspase- 3 activity was significantly increased in the TUG1 KD + HG group compared with the $\mathrm{WT}+\mathrm{HG}$ group (Fig. 2C). The $\mathrm{Bax} / \mathrm{Bcl} 2$ protein expression ratio was significantly increased in the TUG1 KD + HG group compared with the WT + HG group (Fig. 2D and E). Taken together, 

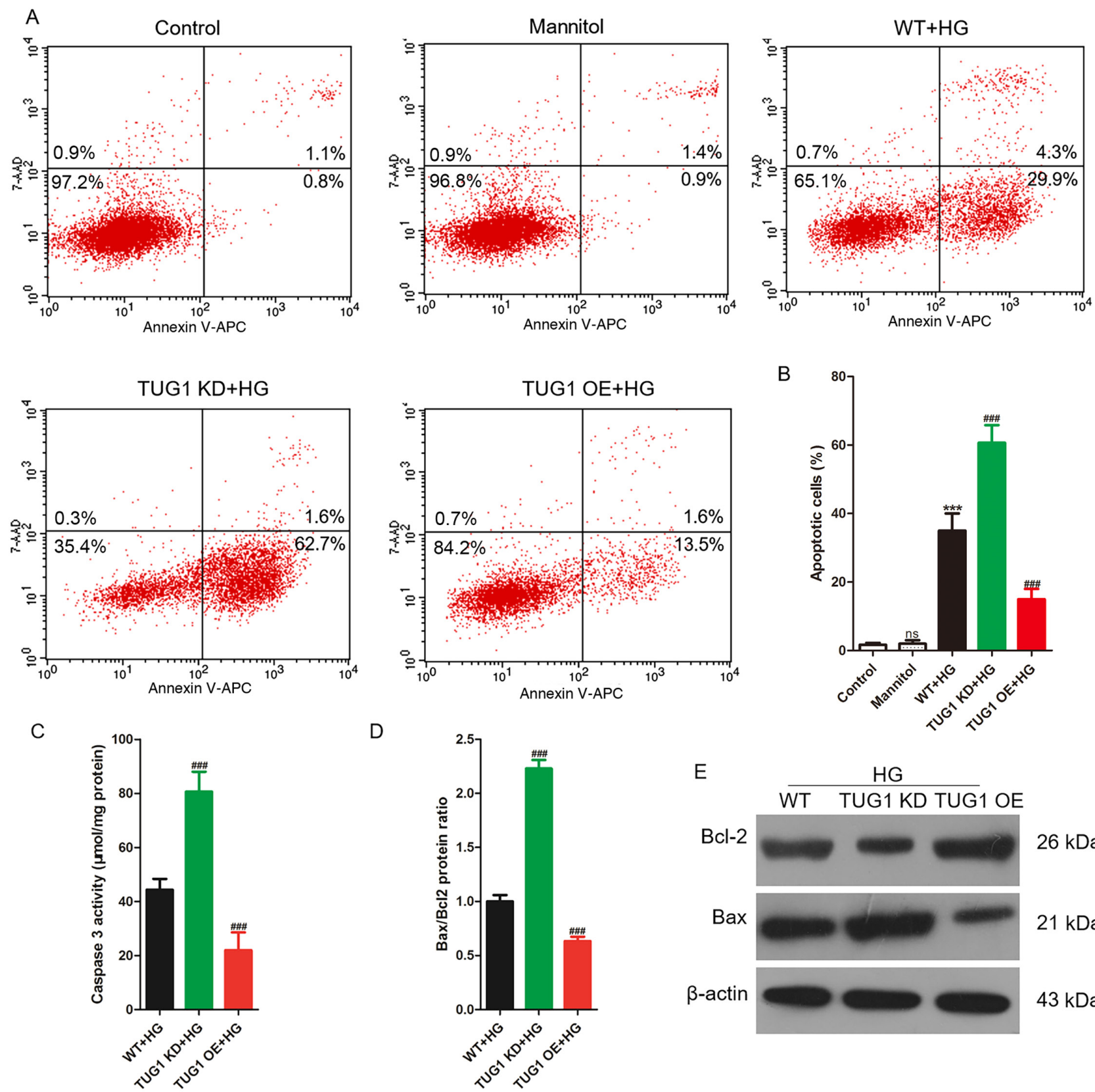

$26 \mathrm{kDa}$

$21 \mathrm{kDa}$

$43 \mathrm{kDa}$

Figure 2. TUG1 KD increases and TUG1 OE reduces HG-induced apoptosis in mouse podocyte clonal cells. After cells were stimulated with $35 \mathrm{mM}$ glucose for $48 \mathrm{~h}$, flow cytometry was used to detect apoptosis. (A) Representative flow analysis plots and (B) statistical analysis of apoptosis. After harvesting of podocytes, (C) caspase-3 activity was examined, and (D) Bax/Bcl2 protein expression was assessed by (E) western blotting. Each experiment was repeated at least three times, and data are presented as the mean \pm SD. P-values were calculated using one-way ANOVA followed by Tukey's post hoc test. ${ }^{\text {ns }} \mathrm{P}>0.05$ and ${ }^{* * * *} \mathrm{P}<0.001 \mathrm{vs}$. control group; ${ }^{\# \#} \mathrm{P}<0.001$ vs. WT + HG group. TUG1, taurine-upregulated gene 1; KD, knockdown; OE, overexpression; WT, wild-type; HG, high glucose.

TUG1 KD aggravated the effects of HG in podocytes, while TUG1 OE reversed these effects.

TUG1 restores $H G$-induced mitochondrial dysfunction. Caspase-3, Bax and Bcl2 are key molecules in the mitochondrial-mediated apoptosis pathway (21). Additionally, a previous study has indicated that TUG1 regulates mitochondrial bioenergetics in diabetic nephropathy (19). Therefore, the effect of TUG1 expression on mitochondrial bioenergetics was assessed in podocytes stimulated with HG. First, the levels of reactive oxygen species (ROS) were measured in podocytes using the MitoSOX $^{\text {TM }}$ kit, since ROS is the direct cause of lipid peroxidation damage to biofilms and macromolecular substances $(22,23)$.
It was revealed that HG triggered a significant increase in ROS levels in podocytes, which was aggravated by TUG1 KD and reversed by TUG1 OE (Fig. 3A and B). Compared with the control group, $\mathrm{HG}$ treatment was associated with a significant decrease in the levels of complex I and III activity and of basal and maximal OCR. TUG1 KD aggravated this decrease, while TUG1 OE reversed these effects (Fig. 3C-F). Therefore, the present data suggested that $\mathrm{HG}$ induced mitochondrial dysfunction in podocytes, TUG1 KD aggravated this dysfunction and TUG1 OE reversed it.

TUG1 upregulates SIRT1 expression by sponging miR-9. As a non-coding RNA (ncRNA), TUG1 can only participate in 

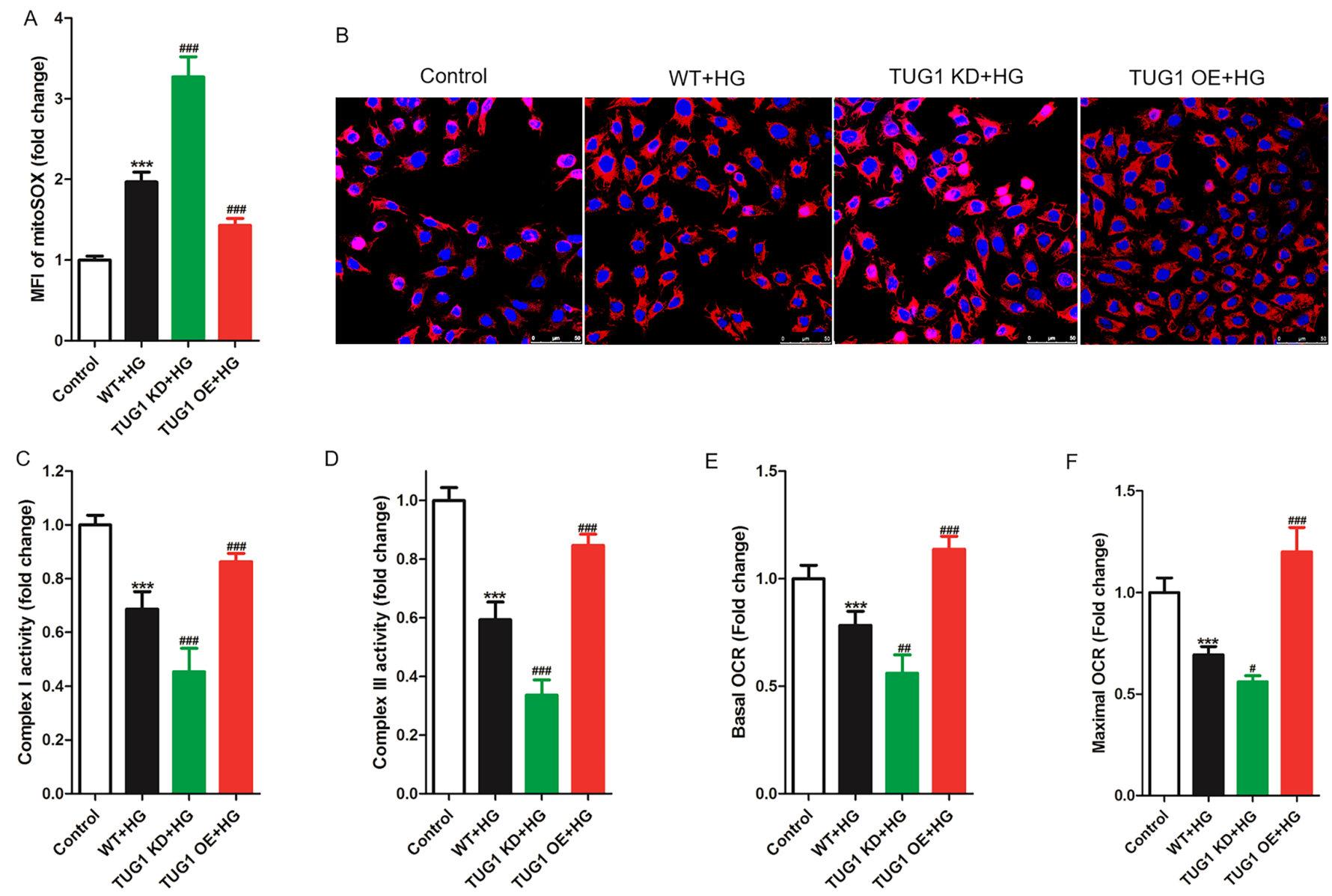

Figure 3. TUG1 modulates mitochondrial bioenergetics in HG-induced mouse podocyte clonal cells. Podocytes were incubated with 35 mM glucose for 48 h. (A) Mitochondrial superoxide production calculated from (B) MitoSOX ${ }^{\mathrm{TM}}$ staining (magnification, x800). Measurement of (C) complex I and (D) complex III activity in mitochondria isolated from cultured podocytes. Seahorse XF24 was used to analyze the (E) basal and (F) maximal OCR in podocytes. Each experiment was repeated at least three times, and data are presented as the mean \pm SD. P-values were calculated using one-way ANOVA followed by Tukey's post hoc test, or Student's t-test accordingly (compared with control group). ${ }^{* * *} \mathrm{P}<0.001$ vs. control group; ${ }^{\#} \mathrm{P}<0.05,{ }^{\# \#} \mathrm{P}<0.01$ and ${ }^{\# \# \#} \mathrm{P}<0.001 \mathrm{vs}$. WT $+\mathrm{HG}$ group. MFI, medial fluorescence intensity; OCR, oxygen consumption rate; TUG1, taurine-upregulated gene 1; KD, knockdown; OE, overexpression; WT, wild-type; HG, high glucose.

the regulation of cell life activities by regulating the expression of downstream genes (10,11). After reviewing a number of previous reports (10-13), miR-9 and SIRT1 were selected as potential downstream genes for TUG1. TUG1 interacts with miR-9 to reduce doxorubicin-induced breast cancer cell apoptosis (24), and the miR-9/SIRT1 axis has been reported to be involved in regulating podocyte damage induced by HG (25). Additionally, resveratrol, an activator of SIRT1, has been demonstrated to improve mitochondrial function by upregulating SIRT1 $(26,27)$. Therefore, it was hypothesized that TUG1 regulated HG-induced apoptosis and mitochondrial function in podocytes via the miR-9/SIRT1 axis.

To examine this hypothesis, miR-9 was overexpressed (mimic) or inhibited (inhibitor) in podocytes (Fig. S2). The potential binding sequences of miR-9/TUG1 and miR-9/SIRT1 are presented in Fig. 4A. WT or MUT binding sequences of TUG1/SIRT1 were co-transfected into podocytes with NC, miR-9-mimic (mimic) or miR-9-inhibitor (inhibitor), and luciferase activity was measured. The results indicated that miR-9 OE significantly reduced, and downregulating miR-9 significantly increased luciferase activity in podocytes after co-transfection with the WT sequence of TUG1/SIRT1 into podocytes (Fig. 4B and C). In addition, RT-qPCR analysis indicated that miR-9 OE significantly reduced, while its inhibition significantly increased, the expression levels of TUG1 and SIRT1 mRNA in podocytes (Fig. 4D). In addition, TUG1 KD significantly reduced the mRNA expression levels of SIRT1 and increased miR-9 expression, while TUG1 OE significantly increased the mRNA expression levels of SIRT1 and reduced miR-9 expression in podocytes (Fig. 4E). Inhibiting miR-9 reversed the inhibition of SIRT1 protein expression induced by TUG1 KD, and miR-9 OE also reversed the promotion of SIRT1 protein expression induced by TUG1 OE (Fig. 4F). Taken together, these results indicated that TUG1 upregulated SIRT1 expression by sponging miR-9 in podocytes.

TUG1 protects $H G$-induced podocytes by upregulating SIRT1. Based on the aforementioned data and previous studies $(26,27)$, it was hypothesized that TUG1 protected podocytes from HG-induced apoptosis and mitochondrial dysfunction by upregulating SIRT1. To test this hypothesis, AAVs were used to infect TUG1 KD podocytes in order to overexpress SIRT1 (SIRT1 OE; Fig. S3). After stimulation with $35 \mathrm{mM}$ glucose for $48 \mathrm{~h}$, podocytes were harvested to analyze apoptosis using flow cytometry. The results revealed that, compared with the TUG1 KD group, the percentage of apoptotic podocytes in TUG1 KD + SIRT1 OE group was significantly decreased (Fig. 5A and B). In addition, the levels 
A
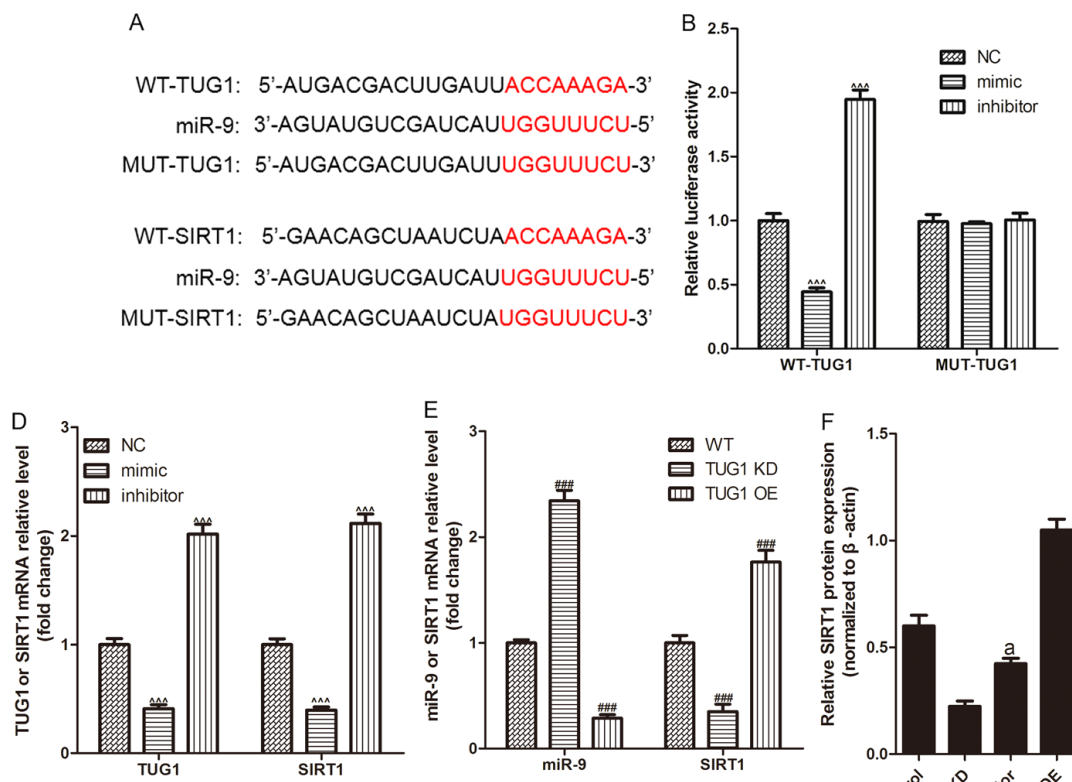

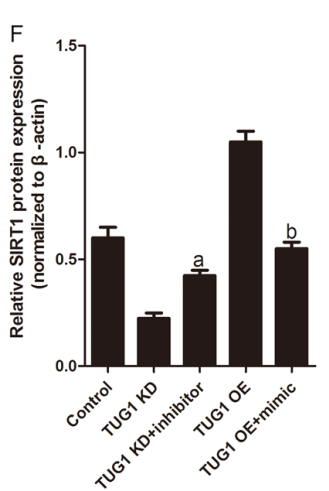

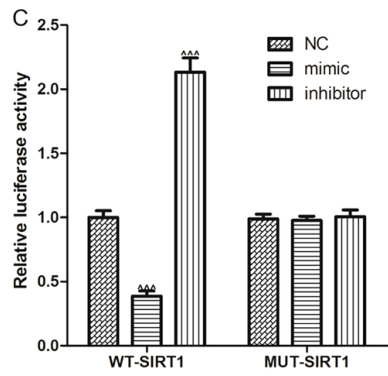

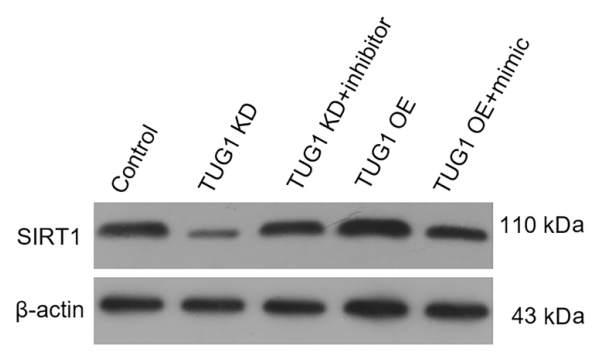

Figure 4. TUG1 promotes SIRT1 expression by inhibiting miR-9 in mouse podocyte clonal cells. (A) Predicted binding sequence of miR-9/TUG1 and miR-9/ SIRT1, including WT and MUT types. WT or MUT sequence of (B) TUG1 or (C) SIRT1 which was predicted to bind to miR-9 were co-transfected into podocytes with NC, mimic or inhibitor, and then luciferase activity was measured. mRNA expression levels of (D) TUG1 and SIRT1 after transfection with miR-9 mimic or inhibitor, and (E) miR-9 and SIRT1 after transfection of TUG1 KD or OE, as determined by reverse transcription-quantitative PCR. (F) Western blotting was used to detect the protein expression levels of SIRT1 in podocytes treated with TUG1 KD, TUG1 KD + miR-9 inhibitor, TUG1 OE and TUG1 $\mathrm{OE}+$ miR-9 mimic. Each experiment was repeated at least three times, and data are presented as the mean $\pm \mathrm{SD}$. P-values were calculated using one-way ANOVA followed by Tukey's post hoc test, or Student's t-test accordingly. ${ }^{\wedge \wedge} \mathrm{P}<0.001 \mathrm{vs}$. NC group; ${ }^{\# \# \# ~} \mathrm{P}<0.001 \mathrm{vs}$. WT group; ${ }^{\text {a }}<0.001$ vs. TUG1 KD group; ${ }^{\text {b}} \mathrm{P}<0.001$ vs. TUG1 OE group. miR, microRNA; SIRT1, sirtuin 1; TUG1, taurine-upregulated gene 1; KD, knockdown; OE, overexpression; WT, wild-type; MUT, mutated; HG, high glucose; NC, negative control.

of MitoSOX in the TUG1 KD + SIRT1 OE group were significantly lower than those in the TUG1 KD group (Fig. S4). The levels of complex I and III activity, and basal and maximal OCR in the TUG1 KD + SIRT1 OE group were significantly higher than those in the TUG1 KD group (Fig. 5C).

\section{Discussion}

A total of $75 \%$ of human genomic DNA is transcribed into RNA, but only $2 \%$ of RNA encode proteins, and $98 \%$ of transcripts are ncRNAs $(28,29)$. Single-stranded RNAs with a length of 20-24 nucleotides are called non-coding single-stranded RNA molecules, while an ncRNA with a length of $>200$ nucleotides is called a lncRNA $(28,29)$. IncRNAs were originally considered to be 'junk' RNA, but previous research has indicated that IncRNAs serve an important role in several activities, such as the dose compensation effect in which the genetic effects of the same phenotype appear in individuals with two or more copies of the gene and individuals with only one gene (30), as well as epigenetic, cell cycle and cell differentiation regulation $(17,31)$. TUG1 lncRNA has been reported to be related to $\operatorname{DKD}(8,32)$.

In the present study, an in vitro HG podocyte damage model was established and TUG1 expression was quantified using RT-qPCR. The present results indicated that HG decreased TUG1 expression in a dose-dependent manner. TUG1 KD aggravated, and TUG1 OE reversed the apoptosis caused by HG in podocytes, suggesting that TUG1 protected podocytes from HG-induced apoptosis. Consistent with the present results, a previous study linked TUG1 with HG-induced podocyte damage, Lei et al (33) reported that the expression levels of TUG1 were decreased in DKD tissues and HG-treated podocytes, and that TUG1 could alleviate HG-induced podocyte apoptosis in vitro. Additionally, TUG1 could be used as a drug target to attenuate podocyte apoptosis and protect diabetic rats from DKD (34). Taken together, the previous studies and the present results demonstrated that TUG1 protected podocytes from HG-induced apoptosis.

The maintenance of normal functions of all cells is closely related to mitochondria. Mitochondria carry out aerobic respiration, and they participate in the processes of cell proliferation, differentiation, apoptosis and information transmission between cells (34). Podocyte damage is a key hallmark of DKD progression, and the peculiarities of the structure and function of the multi-stage foot process of the podocyte make it an energy-intensive cell (2). A large number of mitochondria can be found in both the foot processes and cell bodies of podocytes, and most of the energy required for the cell to function normally is obtained through oxidative phosphorylation in mitochondria; as a result, mitochondrial dysfunction serves an important role in podocyte damage in $\operatorname{DKD}(3,4)$. In addition, TUG1 has been reported to be involved in the regulation of mitochondrial bioenergetics in diabetic nephropathy (7). In the present study, the effect of TUG1 expression on mitochondrial bioenergetics of podocytes was assessed following HG stimulation. The present results indicated that HG induced mitochondrial dysfunction in podocytes, TUG1 KD aggravated this dysfunction and TUG1 OE reversed it. 
A
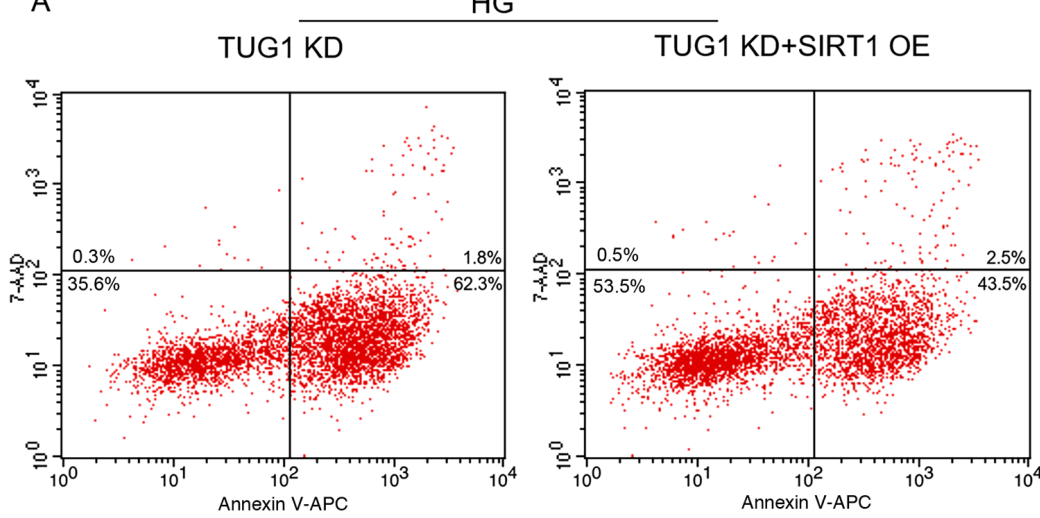

C

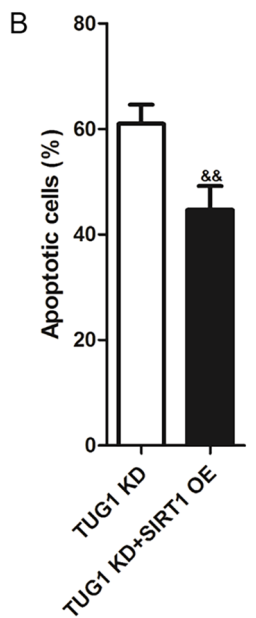

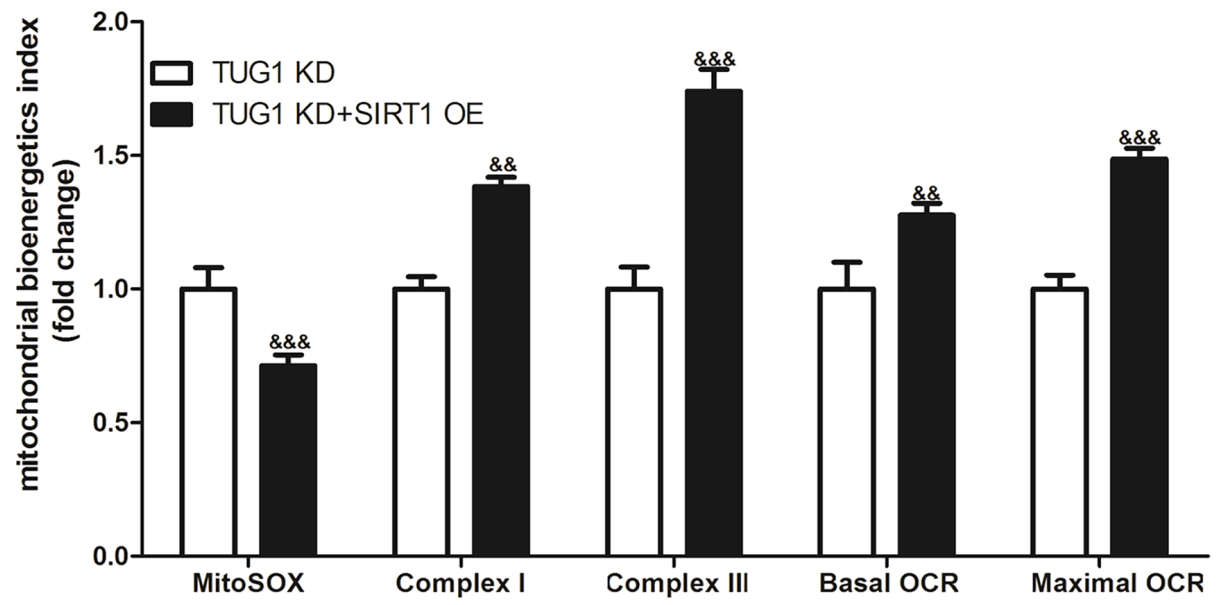

Figure 5. SIRT1 OE reverses HG-induced apoptosis and mitochondrial dysfunction exacerbated by TUG1 KD in mouse podocyte clonal cells. After podocytes were incubated with $35 \mathrm{mM}$ glucose for $48 \mathrm{~h}$, apoptosis was detected using flow cytometry. (A) Representative flow cytometry analysis plots and (B) statistical analysis of apoptosis. (C) Levels of mitochondrial superoxide production, complex I and III activities, and basal and maximal OCR were determined to assess mitochondrial function. Each experiment was repeated at least three times, and data are presented as the mean \pm SD. P-values were calculated using an unpaired Student's t-test. ${ }^{\& \&} \mathrm{P}<0.01$ and ${ }^{\& \& \&} \mathrm{P}<0.001$ vs. TUG1 KD group. SIRT1, sirtuin 1; TUG1, taurine-upregulated gene 1; KD, knockdown; OE, overexpression; HG, high glucose; OCR, oxygen consumption rate.

As a lncRNA, TUG1 is not translated into a protein. Therefore, regulatory genes downstream of TUG1 were selected to study the molecular mechanisms regulating HG-induced mitochondrial dysfunction and apoptosis in podocytes. In the present study, miR-9 and SIRT1 were selected as potential downstream genes for TUG1, as numerous previous studies have reported associations between TUG1, miR-9, SIRT1 and mitochondrial function (21-24). The present study reported that TUG1 promoted SIRT1 expression by sponging miR-9, and SIRT1 OE reversed the HG-induced apoptosis and mitochondrial dysfunction worsened by TUG1 KD.

In the present study, HG decreased TUG1 expression in a dose-dependent manner, and TUG1 OE reversed the mitochondrial dysfunction and apoptosis caused by HG in podocytes. In addition, TUG1 was revealed to upregulate SIRT1 expression through sponging miR-9, and SIRT1 OE decreased the apoptosis in HG-induced TUG1 KD podocytes. In conclusion, it was revealed that IncRNA TUG1 regulated HG-induced apoptosis and mitochondrial dysfunction via the miR-9/SIRT1 axis, and this revealed a molecular mechanism of podocyte apoptosis and mitochondrial dysfunction regulation, and provided potential targets for diabetic nephropathy prevention and treatment.

\section{Acknowledgements}

Not applicable.

\section{Funding}

No funding was received.

\section{Availability of data and materials}

The datasets used and/or analyzed during the current study are available from the corresponding author on reasonable request.

\section{Authors' contributions}

YH made substantial contributions to the conception and design of the present study. ML, GK, YW and DL acquired the data, and analyzed and interpreted the data. ML and $\mathrm{YH}$ drafted the article and revised it critically for important intellectual content. All authors agreed to submit to the current journal, and agreed to be accountable for all aspects of the work. ML and YH confirm the authenticity of all the raw data. All authors have read and approved the final manuscript. 


\section{Ethics approval and consent to participate}

Not applicable.

\section{Patient consent for publication}

Not applicable.

\section{Competing interests}

The authors declare that they have no competing interests.

\section{References}

1. Alicic RZ, Rooney MT and Tuttle KR: Diabetic kidney disease: Challenges, progress, and possibilities. Clin J Am Soc Nephrol 12: 2032-2045, 2017.

2. Altintas MM and Reiser J: Podocytes: Way to Go. Am J Pathol 189: 226-228, 2019

3. Lin JS and Susztak KJ: Podocytes: the weakest link in diabetic kidney disease? Curr Diab Rep 16: 45, 2016.

4. Forbes JM and Thorburn DR: Mitochondrial dysfunction in diabetic kidney disease. Nat Rev Nephrol 14: 291-312, 2018.

5. Wei PZ and Szeto CC: Mitochondrial dysfunction in diabetic kidney disease. Clin Chim Acta 496: 108-116, 2019.

6. Piwkowska A, Rogacka D, Jankowski M, Dominiczak MH Stepiński JK and Angielski S: Metformin induces suppression of NAD $(\mathrm{P}) \mathrm{H}$ oxidase activity in podocytes. Biochem Biophys Res Commun 393: 268-273, 2010

7. Lee SH, Moon SJ, Paeng J, Kang HY, Nam BY, Kim S, Kim CH, Lee MJ, Oh HJ, Park JT, et al: Podocyte hypertrophy precedes apoptosis under experimental diabetic conditions. Apoptosis 20: 1056-1071, 2015.

8. Long J, Badal SS, Ye Z, Wang Y, Ayanga BA, Galvan DL, Green NH, Chang BH, Overbeek PA and Danesh FR: Long noncoding RNA Tug1 regulates mitochondrial bioenergetics in diabetic nephropathy. J Clin Invest 126: 4205-4218, 2016.

9. Yue P, Jing L, Zhao X, Zhu H and Teng J: Down-regulation of taurine-up-regulated gene 1 attenuates inflammation by sponging miR-9-5p via targeting NF- $\kappa \mathrm{B} 1 / \mathrm{p} 50$ in multiple sclerosis. Life Sci 233: 116731, 2019

10. Hong Q, Zhang L, Das B, Li Z, Liu B, Cai G, Chen X, Chuang PY, $\mathrm{He} \mathrm{JC}$ and Lee K: Increased podocyte Sirtuin-1 function attenuates diabetic kidney injury. Kidney Int 93: 1330-1343, 2018

11. Zhang Y, Chang B, Zhang J and Wu X: LncRNA SOX2OT alleviates the high glucose-induced podocytes injury through autophagy induction by the miR-9/SIRT1 axis. Exp Mol Pathol 110: 104283,2019

12. Cai X, Bao L, Ren J, Li Y and Zhang Z: Grape seed procyanidin $\mathrm{B} 2$ protects podocytes from high glucose-induced mitochondrial dysfunction and apoptosis via the AMPK-SIRT1-PGC- $1 \alpha$ axis in vitro. Food Funct 7, 805-815, 2016.

13. Lei X, Zhang L, Li Z and Ren J: Astragaloside IV/lncRNA-TUG1/ TRAF5 signaling pathway participates in podocyte apoptosis of diabetic nephropathy rats. Drug Des Devel Ther 12: 2785-2793, 2018.

14. Chen J, Gui D, Chen Y, Mou L, Liu Y and Huang J: Astragaloside IV improves high glucose-induced podocyte adhesion dysfunction via alpha3beta1 integrin upregulation and integrin-linked kinase inhibition. Biochem Pharmacol 76: 796-804, 2008.

15. Livak KJ and Schmittgen TD: Analysis of relative gene expression data using real-time quantitative PCR and the $2(-\Delta \Delta \mathrm{C}(\mathrm{T}))$ method . Methods 25: 402-408, 2001.

16. Chen ZZ, Huang L, Wu YH, Zhai WJ and Gao YF: LncSox4 promotes the self-renewal of liver tumour-initiating cells through Stat3-mediated Sox4 expression. Nat Commun 7: 12598, 2016.
17. Jie Tao J, Zhang J, Ling Y, McCall CE and Liu TF: Mitochondrial Sirtuin 4 resolves immune tolerance in monocytes by rebalancing glycolysis and glucose oxidation homeostasis. Front Immunol 9: 419, 2018

18. Long J, Badal SS, Ye Z, Wang Y, Ayanga BA, Galvan DL, Green NH, Chang BH, Overbeek PA and Danesh FR: Long noncoding RNA Tug1 regulates mitochondrial bioenergetics in diabetic nephropathy. J Clin Invest 126: 4205-4218, 2016.

19. Maestroni S and Zerbini G: Glomerular endothelial cells versus podocytes as the cellular target in diabetic nephropathy. Acta Diabetol 55: 1105-1111, 2018.

20. Shang X, Ji X, Dang J, Wang L, Sun C, Liu K, Sik A and Jin M: $\alpha$-asarone induces cardiac defects and QT prolongation through mitochondrial apoptosis pathway in zebrafish. Toxicol Lett 324: $1-11,2020$.

21. Yang B, Chen Y and Shi J: Reactive oxygen species (ROS)-based nanomedicine. Chem Rev 119: 4881-4985, 2019.

22. Turrens JF: Mitochondrial formation of reactive oxygen species. J Physiol 552: 335-344, 2003

23. Wang S, Cheng M, Zheng X, Zheng L, Liu H, Lu J, Liu Y and Chen W: Interactions between IncRNA TUG1 and miR-9 modulate the resistance of breast cancer cells to doxorubicin by regulating eIF5A2. Onco Targets Ther 13: 13159-13170, 2020.

24. Zhang Y, Chang B, Zhang J and Wu X: LncRNA SOX2OT alleviates the high glucose-induced podocytes injury through autophagy induction by the miR-9/SIRT1 axis. Exp Mol Pathol 110: $104283,2019$.

25. Lagouge M, Argmann C, Gerhart-Hines Z, Meziane H, Lerin C, Daussin F, Messadeq N, Milne J, Lambert P, Elliott P, et al: Resveratrol improves mitochondrial function and protects against metabolic disease by activating SIRT1 and PGC-1 $\alpha$. Cell 127: 1109-1122, 2006.

26. Yousuf S, Atif F, Ahmad M, Hoda N, Ishrat T, Khan B and Islam F: Resveratrol exerts its neuroprotective effect by modulating mitochondrial dysfunctions and associated cell death during cerebral ischemia. Brain Res 1250: 242-253, 2009.

27. Zhao Y, Li H, Fang S, Kang Y, Wu W, Hao Y, Li Z, Bu D, Sun N, Zhang MQ, et al: NONCODE 2016: an informative and valuable data source of long non-coding RNAs. Nucleic Acids Res 44: D203-D208, 2016

28. Ulitsky I: Evolution to the rescue: using comparative genomics to understand long non-coding RNAs. Nat Rev Genet 17: 601-614, 2016.

29. Militti C, Maenner S, Becker PB and Gebauer F: UNR facilitates the interaction of MLE with the lncRNA roX2 during Drosophila dosage compensation. Nat Commun 5: 4762, 2014

30. Rinn JL and Chang HY: Long noncoding RNAs: molecular modalities to organismal functions. Annu Rev Biochem89: 283-308, 2020

31. Li SY and Susztak K: The long noncoding RNA Tug1 connects metabolic changes with kidney disease in podocytes. J Clin Invest 126: 4072-4075, 2016.

32. Li Y, Huang D, Zheng L, Cao H, Gao Y, Yanga Y and Fan Z: Long non-coding RNA TUG1 alleviates high glucose induced podocyte inflammation, fibrosis and apoptosis in diabetic nephropathy via targeting the miR-27a-3p/E2F3 axis. RSC Adv 9: 37620-37629, 2019.

33. Lei X, Zhang L, Li Z and Ren J: Astragaloside IV/lncRNA-TUG1/ TRAF5 signaling pathway participates in podocyte apoptosis of diabetic nephropathy rats. Drug Des Devel Ther 12: 2785-2793, 2018.

34. Roger AJ, Muñoz-Gómez SA and Kamikawa R: The origin and diversification of mitochondria. Curr Biol 27: R1177-R1192, 2017.

(i) 9 This work is licensed under a Creative Commons Attribution-NonCommercial-NoDerivatives 4.0 International (CC BY-NC-ND 4.0) License. 